\title{
Target Detection using DLR Earth Sensing Imaging Spectrometer (DESIS) data
}

\author{
Arati Paul ${ }^{1}$, Dibyendu Dutta ${ }^{1}$ and C. S. Jha ${ }^{2}$ \\ 1 Regional Remote Sensing Centre-East, NRSC, ISRO, Kolkata, India \\ 2 National Remote Sensing Centre, ISRO, Hyderabad, India
}

Commission III, WG III/4

KEY WORDS: Target detection, DESIS, ACE, Unmixing, brick kilns, Photovoltaic panels.

\begin{abstract}
:
DLR's Earth Sensing Imaging Spectrometer (DESIS) is mounted on the International Space Station (ISS). DESIS records data in the spectral range from 400 to $1000 \mathrm{~nm}$ with a spectral and spatial resolution of $2.55 \mathrm{~nm}$ and $30 \mathrm{~m}$ respectively. The high spectral resolution enables in detecting a target object distinctly in remotely sensed imagery which has many useful applications in different fields of surveillance and monitoring. In present work two different case studies have been carried out that use DESIS data for target detection. In the first case study brick kilns are detected in DESIS data using Adaptive Coherence Estimator (ACE) algorithm. In the second case study Photovoltaic (PV) panels are considered as target object and linear spectral unmixing is employed to distinctly detect them in the image. From experimental results it is observed that the first target which were sparsely located in the image is detected very precisely with F1 score value of 0.97 . The accuracy of the output of PV panel detection is observed to be more than $98 \%$. Both the case studies show the potential of DESIS data in target detection which is a very important application of hyperspectral remote sensing.
\end{abstract}

\section{INTRODUCTION}

Hyperspectral image with its rich spectral information has found many applications in various fields (Paul et al.; 20215), such as astronomy, agriculture (Datt et al., 2003), mineralogy (Hörig et al., 2001), military (Eismann et al., 2009), and in particular, target detection (Manolakis et al., 2014; Frontera-Pons et al., 2017, Cavalli et al., 2013). The concept of target detection is to find out whether a pixel is occupied (fully or partially) by the target material or it belongs to the background (Bitar et al., 2020).

Hyperspectral sensors capture the reflective (or emissive) characteristics of objects in the visible to infrared (IR) regions of the spectrum (Paul et al., 2015; Paul and Chaki, 2020). The huge spectral dimension of hyperspectral image is generally reduced to obtain better classification accuracy in certain applications (Paul and Chaki, 2021a; Paul and Bhoumik, 2021; Paul et al., 2021a). In addition to dimensionality reduction (Paul and Chaki, 2019; Paul and Chaki, 2021b), hyperspectral images are also enhanced for noise reduction and better representation (Paul et al., 2021b). By utilising the spectral signatures of the materials hyperspectral data enables algorithms to detect targets of interest in a hyperspectral scene. Hence, target detection is basically a binary classification that labels each pixel in the image either as a target or background.

Target detection algorithms work in supervised as well as in unsupervised way. The unsupervised way of target detection is also known as anomaly detection where no prior knowledge of the target spectral signature is used or assumed. Anomaly detectors uses statistical measures to detect objects that are distinct from the cluttered background. For instance, pixels that have a significantly different spectral signature from their neighboring background pixels are identified as spectral anomalies (Matteoli et al., 2010). Reed-Xiaoli (RX) anomaly detector (Reed and Yu, 1990) is a classic example of this kind of algorithms that has been successfully applied in many hyperspectral target detection applications.
In contrast to the anomaly detection, in the supervised target detection, prior knowledge about the spectral characteristics of the desired targets is available. Hence it is also known as signature-based target detection. In this case, a single target spectrum (Robey et al., 1992) or a target subspace (Scharf and Friedlander, 1994) is used to define the spectral characteristics of the target object. The local/ global background is modelled by a Gaussian distribution or by subspace statistics (Nasrabadi, 2014).

In present work supervised target detection has been carried out on DESIS datasets. Two case studies have been demonstrated here. In first case study, the target object is brick kiln whereas solar panel is considered as target object in the second case study. Brick kiln is a thermally insulated chamber where blocks of clay are baked into bricks. It has been a focus of researchers from decades for many reasons. Apart from the business (eBrickkiln, 2020), industry (Kiln Eco, 2015) and technology (Maithel et al., 2014, beralmar, 2011) related reasons, brick kilns are also studied for environmental pollution (Weyant et al., 2014) and labour related activities (Boyd et al., 2018). Brick kiln industry requires to be closely monitored by the administration and stake holders. With the help of increasing spectral resolution, hyperspectral data has a potential to detect brick kiln locations efficiently. The spectral patterns of target objects are extracted from the scene for known locations. Adaptive coherence estimator (ACE) algorithm (Bar et al., 2013) is used to detect the target pixels in other locations of the scene.

In recent years, renewable energy systems are growing steadily (Malof et al., 2016; Puttemans et al., 2016; Chang, 2013). An important component of the renewable energy systems is Photovoltaic (PV) panels (or solar panel) that generate greener (i.e., non-polluting) electrical power from solar energy. As this industry is growing, the detailed information concerning these solar systems including their localization are gaining importance (Malof et al., 2016). Remote sensing provides faster and more reliable means of obtaining such precise information (Malof et al., 2016; Bioucas-Dias et al., 2012). Very high-spatial-resolution airborne/spaceborne images with a small number of spectral bands ignore the material properties due to limited number of spectral bands. Hence, very efficient detection is restricted with 
this type of data. In some cases, the visual characteristics of the PV panels get altered due to specular reflections, which makes the detection more difficult (Karoui et al., 2018). On the other hand, hundreds of narrow and contiguous spectral bands of hypersepctral imagery enable more accurate material detection (Cichocki et al., 2009). Each observed pixel-spectrum in an image is compared with reference material spectra by using an appropriate criterion or method to detect the target pixels from in a hyperspectral image (Limem et al., 2013). In the present study DESIS hyperspectral data is used for detecting Photovoltaic panels in the scene using spectral unmixing.

The rest of the article is arranged as follows: The dataset description is given in section 2 whereas the methodology is described in section 3. Section 4 includes the experimental results and final conclusions are drawn in section 5 .

\section{DATA DESCRIPTION AND STUDY AREA}

DLR's Earth Sensing Imaging Spectrometer (DESIS) is mounted on the International Space Station (ISS) (Müller et al., 2016; Krutz et al., 2019). DESIS records data in the spectral range from 400 to $1000 \mathrm{~nm}$ with a spectral resolution of $2.55 \mathrm{~nm}$ and a spatial resolution of $30 \mathrm{~m}$. The tile size is $1024 \times 1024$ pixel corresponding to about $30 \times 30 \mathrm{~km} 2$ on the ground. The available types of DESIS products are summarised in table 1 (Alonso et al., 2019). In present study of target detection DESIS L2A product is used.

\begin{tabular}{|l|l|l|}
\hline $\begin{array}{l}\text { Product } \\
\text { Type }\end{array}$ & Description & $\begin{array}{l}\text { Order } \\
\text { Parameters }\end{array}$ \\
\hline L1B & $\begin{array}{l}\text { Radiometric and sensor specific } \\
\text { corrected data. All metadata attached } \\
\text { for further processing }\end{array}$ & $\begin{array}{l}\text { Spectral } \\
\text { Binning }\end{array}$ \\
\hline L1C & $\begin{array}{l}\text { L1B data ortho-rectified and } \\
\text { resampled to a specified grid using } \\
\text { global SRTM 1 arcsec DEM for } \\
\text { terrain correction using global } \\
\text { Landsat ETM+ references for sensor } \\
\text { model refinement }\end{array}$ & Projection, \\
\hline L2A & $\begin{array}{l}\text { L1C data atmospherically } \\
\text { correctedc(Bottom-of-Atmopshere } \\
\text { reflectance) generating several masks } \\
\text { (water, land, cloud, shadow, ...) }\end{array}$ & Resampling \\
\hline
\end{tabular}

Table 1. DESIS product types and descriptions.

In the first case study where the brick kilns are considered as target object the DESIS data acquired on 24th Feb, 2020 with approximate latitude extent from $29^{\circ} 50^{\prime} 57^{\prime \prime} \mathrm{N}$ to $30^{\circ} 16^{\prime} 11^{\prime \prime} \mathrm{N}$ and longitude extent $75^{\circ} 52^{\prime} 57^{\prime \prime} \mathrm{E}$ to $76^{\circ} 18^{\prime} 56^{\prime \prime} \mathrm{E}$ is used. The area covers parts of Punjab and Hariyana state and includes Bir Gurdialpura Wildlife Sanctuary. The major land use includes agriculture. A partial cloud cover is observed in the scene. In the second case study of solar panel detection, the DESIS data of 05th Mar, 2019 is used. The data is acquired over the state of Rajasthan covering the spatial extent with approximate min-max latitude from $27^{\circ} 14^{\prime} 8 " \mathrm{~N}$ to $27^{\circ} 39^{\prime} 35^{\prime \prime} \mathrm{N}$ and longitude from $71^{\circ}$ $49^{\prime} 42^{\prime \prime} \mathrm{E}$ to $72^{\circ} 15^{\prime} 11$ 'E in Thar Desert. The area has solar irradiation of $5.72 \mathrm{kWh} / \mathrm{m} 2 /$ day (Bhadla Solar Park, 2019) and includes many important solar power plants viz. Bhadla solar park, NTPC solar park etc. in the Jodhpur district of Rajasthan. This area receives many sandstorms every year (Supe et al., 2020)

\section{METHODOLOGY}

The methodology for target detection using DESIS hyperspectral data includes stages like data pre-processing and target detection as described in following subsections.

\subsection{Data pre-processing}

DESIS L2A is a corrected data product as given in table 1 . In addition to onboard measurement and laboratory calibration for geometric correction, DESIS uses image matching with reference data for extraction of Ground Control Points (GCP). However, the geometric accuracy of DESIS data used in the first case study is observed a bit low because of poor image matching. Hence, the geometric corrections of the dataset have been carried out using the LANDSAT data.

\subsection{Target detection using Adaptive Coherence Estimator}

The spectral match-based target detection methods make decisions based on signatures. They distinguish the target pixels from the background by comparing the whole image spectra with the known target spectra. The target detection is carried out by using in-scene target spectra that are extracted for known locations and a library is built. The Adaptive coherence estimator (ACE) algorithm is used to detect the target due to its significant detection performance (Jnawali et al., 2018). It assumes a normal distribution for the background and calculate the background covariance adaptively (Yang et al., 2014). The formula of ACE is given in equation (1).

$$
\begin{aligned}
& D_{A C E}(x) \\
& =\frac{\left[(T-\mu)^{T} \operatorname{Cov}^{-1}(x-\mu)\right]^{2}}{\left[(T-\mu)^{T} \operatorname{Cov}^{-1}(T-\mu)\right]\left[(x-\mu)^{T} \operatorname{Cov}^{-1}(x-\mu)\right]}
\end{aligned}
$$

where, $x$ is the data acquired by the sensors, $\mu$ is the background mean, $C o v$ is the background covariance and $T$ is the characteristics of the target spectra. $D_{A C E}$ becomes maximum while $x=T$, that means the test pixel is the target. However, $D_{A C E}$ becomes minimum for $x=\mu$, that shows the test pixel is belongs to the background. However, in practical scenario the results always cannot reach the ideal condition. So, a detection threshold $\eta_{\text {thr }}$ should be set to evaluate the results as given in equation (2).

$$
x=\left\{\begin{array}{l}
\text { target }, \quad D_{A C E}>\eta_{\mathrm{thr}} \\
\text { background, otherwise }
\end{array}\right.
$$

If the calculated value is bigger than the threshold, $x$ is a target pixel otherwise $x$ is considered as background pixel (Bai et al., 2019).

Since only a small fraction of all the pixels in the image can be labelled as targets, the overall classification accuracy is not a good measure of performance since pixels of interest are sparse (Nasrabadi, 2014). Therefore, in present case study, performance of target detection is measure using precision, recall and F1 score as given in equations (3) to (5) respectively. True positive value indicates the number of correctly detected brick kilns whereas false positive value gives the number of wrongly identified kilns. The false negative value represents the number of brick kilns that were actually present in the scene but could not be detected by the process.

$$
\text { Precision }=\frac{\text { True Positive }}{\text { True Positive }+ \text { False Positive }}
$$




$$
\text { Recall }=\frac{\text { True Positive }}{\text { True Positive }+ \text { False Negative }}
$$

$$
F 1=2 \times \frac{\text { Precision } \times \text { Recall }}{\text { Precision }+ \text { Recall }}
$$

\subsection{Target detection using spectral unmixing}

Effect of mixed pixel is prevalent in low or middle resolution remote sensing images. Hence, spectral unmixing has become a widely adopted technique in remote sensing for quantitative analysis. Linear Spectral Unmixing is used to determine the relative abundance of materials that are captured by hyperspectral imagery based on the materials' spectral characteristics. In present study the linear spectral unmixing is used to detect pixels that contain PV panel in the DESIS data. Here, the reflectance of each material (or endmember) is assumed to be known. Each pixel of the input data is assumed to be a linear combination of the endmembers. Linear Spectral Unmixing is employed to estimate the abundance values of each endmember for every pixel in the scene. It performs inversion of library matrix and multiplication by the observed spectrum as given in equation (6) to (7).

$$
\begin{gathered}
A^{M \times N} * X^{N \times 1}=B^{M \times 1} \\
\text { or } \\
X=A^{-1} * B \quad(7)
\end{gathered}
$$

where, $\mathrm{A}$ is endmember library matrix with $\mathrm{N}$ number of spectrums, B is observed spectrum from data with $M$ number of bands and $\mathrm{X}$ is unknown abundance vector respectively.

The fraction of the pixel that contains the endmember material is provided in the abundance images for the corresponding material. The original observed spectrum is compared with the best fitting spectrum to estimate the error. It's also a good indicator for missing or incorrect endmembers.

\section{RESULT AND DISCUSSION}

Results of target detection using DESIS data for two different target objects are discussed in following subsections.

\subsection{Detection of brick kiln}

In present study the DESIS L2A data is first geometrically rectified using LANDSAT data (figure 1). Next, the target spectrum is generated from the scene for known locations. A sample target spectrum is given in figure 2 .

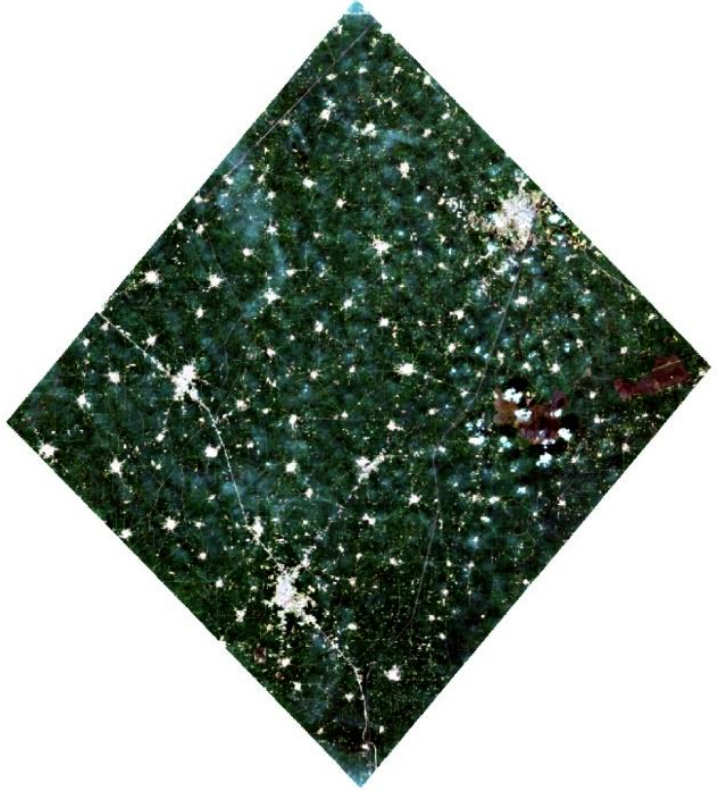

Figure 1: Rectified DESIS data of the study area (RGB bands: $94,59,28)$

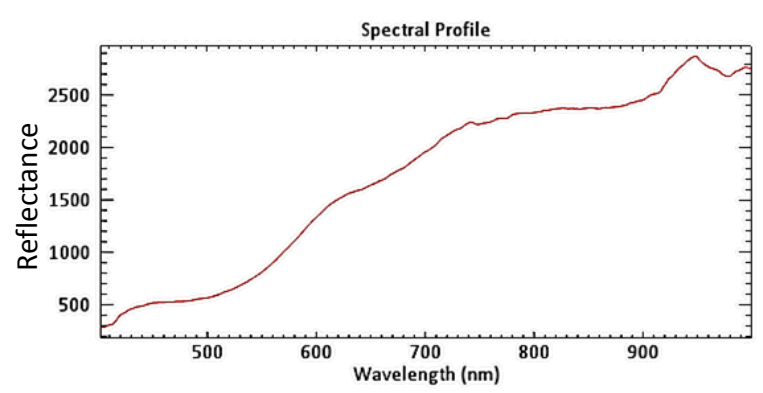

Figure 2: Target spectra of brick kilns

The ACE algorithm is applied on the data to generate target map as described in section 3.1 with threshold value of 0.25 . The target map of brick kiln locations in the scene is given in figure 3a where white spots represent the detected brick kiln's locations. Two such spots are highlighted in red and yellow dots in figure $3 \mathrm{a}$ for which high resolution images are shown in figures $3 \mathrm{~b}$ and $3 \mathrm{c}$ respectively. It is observed in this figure that Brick kilns are detected correctly and this shows the case of true positive detection. 


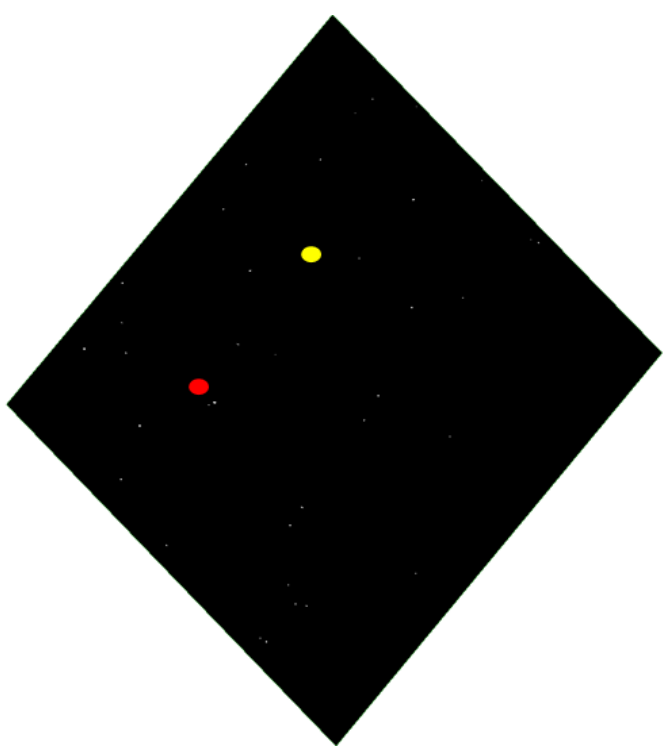

(a)

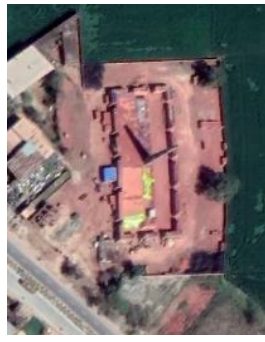

(b)

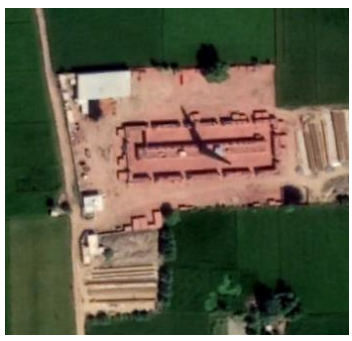

(c)
Figure 3: (a) Target map of brick kilns, HR image of the (b) Red and (c) Yellow marked locations respectively showing true ppositive detections.

The target detection result is verified using high resolution (HR) Google Earth satellite imagery of the same location for performance assessment. The precision, recall and the F1 Score of the output is estimated using equations (3) to (5) respectively. Precision value of 0.96 is observed which is quite high. Precision is the ratio of correctly detected brick kilns to the total detected brick kilns. A high precision value indicates a smaller number of false detections. It is a very important aspect of target detection in a remotely sensed image where background of the target is heterogeneous. Hence, the actual number of true negative points are further analysed. Only 2 numbers of true negative detections are observed in the scene. In first case the target object was covered by cloud and a very different spectral signature is obtained at that location as given in figure $4 \mathrm{a}$. the corresponding scene reference is given in figure $4 \mathrm{bHence}$, it could not be identified by the present method.

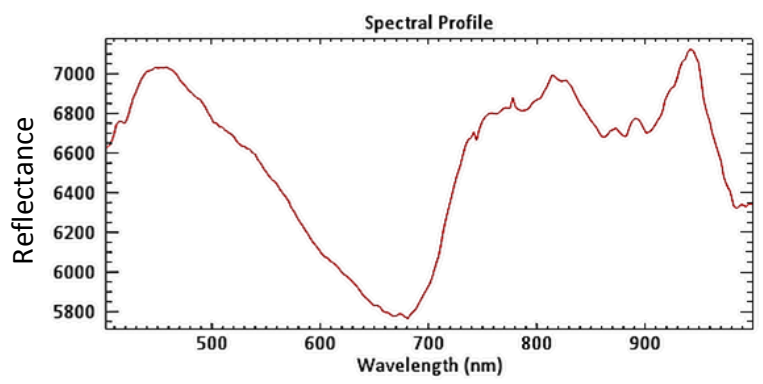

(a)

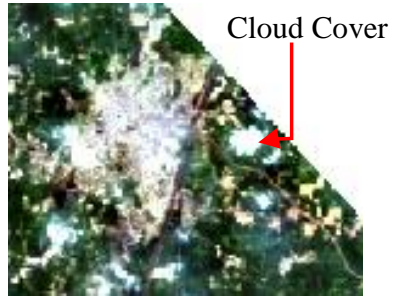

(b)

Figure 4: (a) Spectral signature of the cloud covered target and (b) DESIS Scene reference.

In the second case the target is found to be mostly occupied by vegetation as is mostly the case with abandoned kilns. This made the target to be mixed with the background (figure 5). Hence, the target is not distinctly detected using the present method.

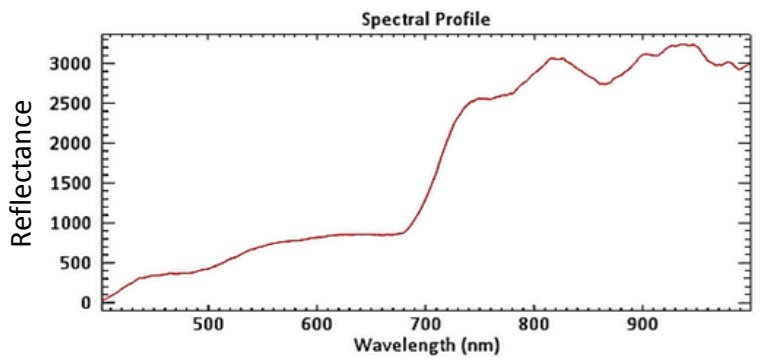

(a)

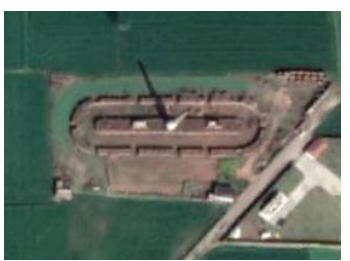

(b)

Figure 5: (a) Spectral signature of the target mixed with vegetation, (b) HR image of the location.

The ACE method also performed well in terms of Recall value, i.e., 0.98 . Recall indicates the sensitivity of the detection method i.e., ratio of the number of correctly detected brick kilns to the total number of brick kilns that are actually present in the scene. A high Recall value indicated a high detection capability. In present study only one such instance is observed in the output where false positive detection has occurred. Although a dissimilarity in spectral signature of the ground object in the wavelength range about $740 \mathrm{~nm}$ to $900 \mathrm{~nm}$ is observed at false positive target location (figure 6) it has very similar response to the target object in the other regions of spectra (figure 2). Additionally, it has a distinct presence with respect to background in the scene. Hence, in present method it is also detected as the target.

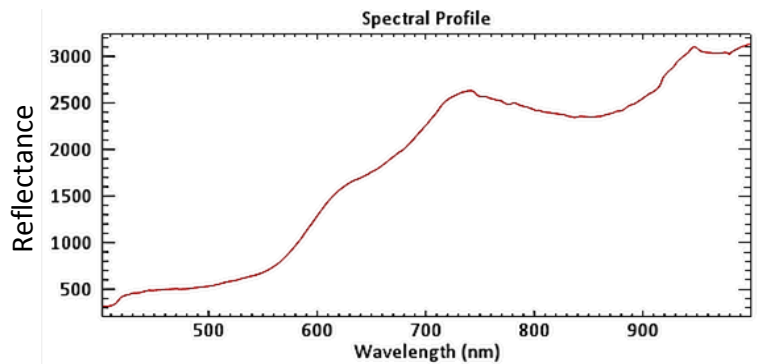

(a) 


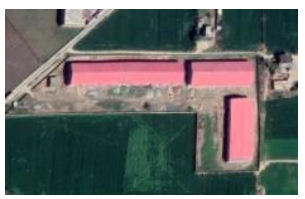

(b)

Figure 6: (a) Spectral signature of ground object at false positive target location, (b) HR image of the location.

F1 Score is the weighted average of Precision and Recall and measures the balance between these two metrics. The value of the F1 score is 0.97 which is quite high. The DESIS data acquired in high spectral resolution enables in achieving this high F1 score compared to what has been reported using multispectral data of higher spatial resolution in the recent past (Nazir et al., 2020; Misra et al., 2020).

\subsection{Detection of solar panel}

A sample target spectrum of photovoltaic panels extracted from the data (figure 7) is given in figure 8 where a high value in the spectral range above $900 \mathrm{~nm}$ is observed and a lower value in about $450-500 \mathrm{~nm}$ region is evident. However, it gives a relatively higher reflectance in bands below $450 \mathrm{~nm}$.

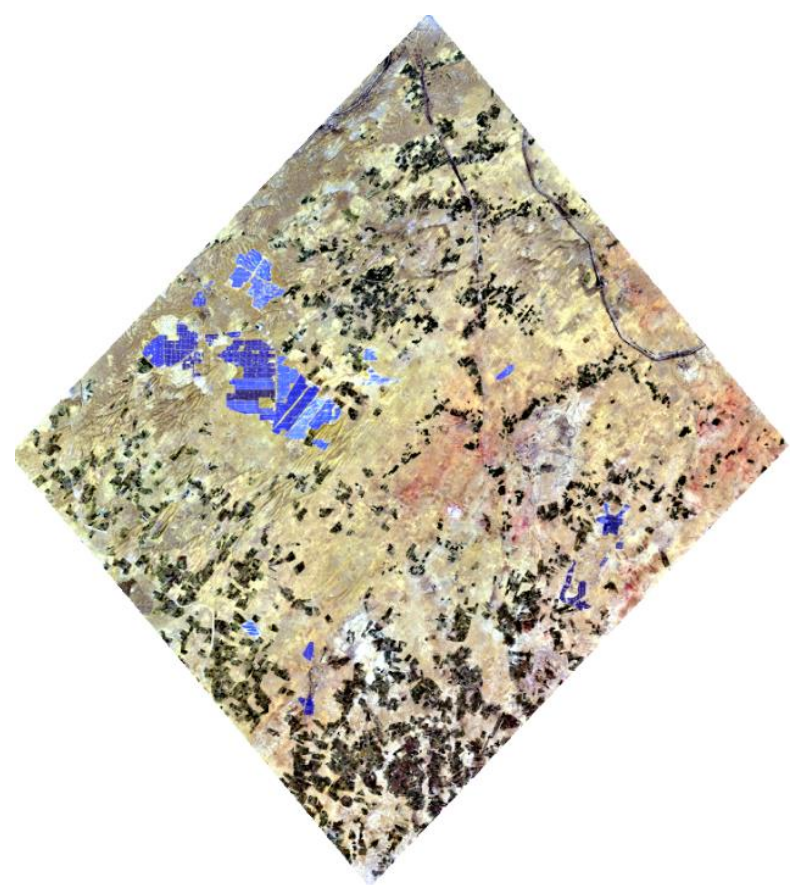

Figure 7: Rectified DESIS data of the study area (RGB: 94, 58, 10).

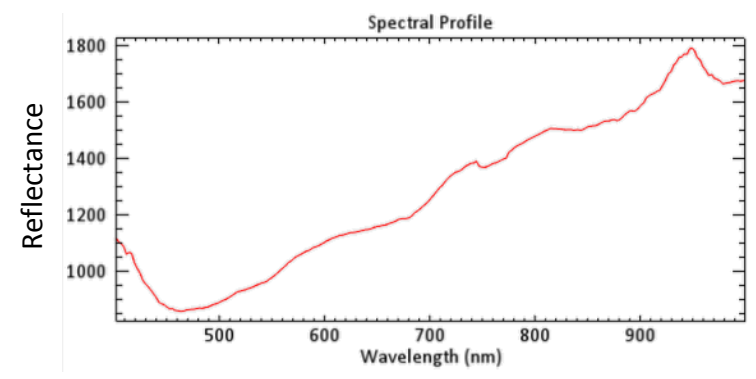

Figure 8: Target spectra of photovoltaic panels.
Three major spectrums of three major land cover types are extracted from the scene that include spectrum of PV panel, vegetation and sand (figure 9). Subsequently linear spectral unmixing is carried out using these spectrums. The result of unmixing is given in figure 10, where the abundance image of vegetation, sand and PV panel are depicted as RGB. The PV panels are clearly visible as blue patches in the image.

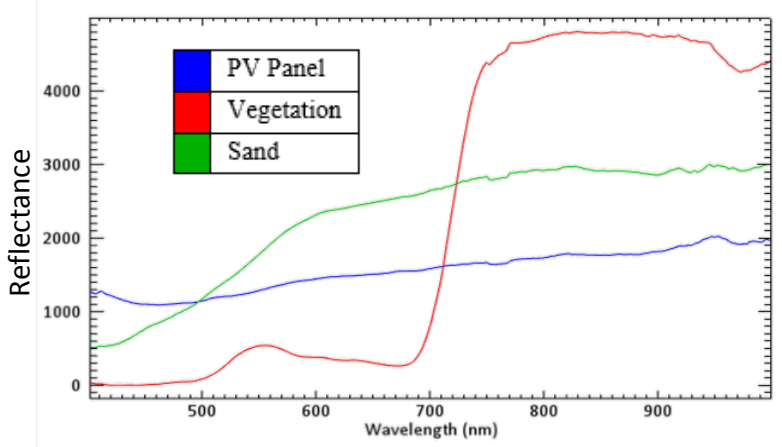

Figure 9: Library spectrum extracted from data for unmixing.

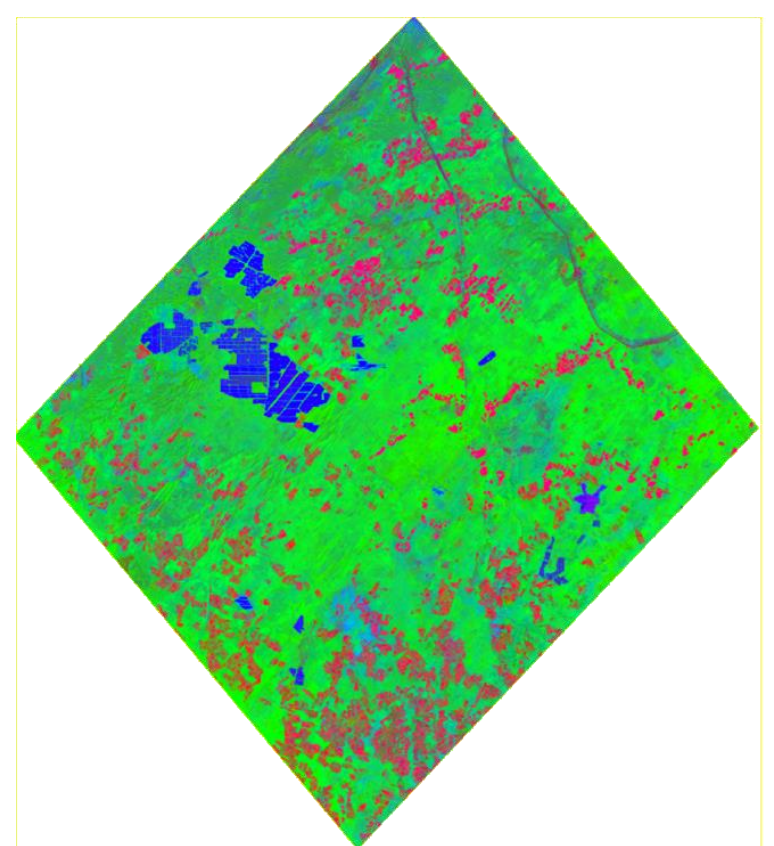

Figure 10: Abundance image of vegetation, sand and PV panel as RGB

Further to extract solar panel pixels from the data a post processing steps are carried out where the PV panel abundance image is thresholded with the value of 0.7 and ANDed with the inverse of thresholded output of sand with the value of 0.5 . Finally, the output is converted into a binary image as depicted in figure 11. 


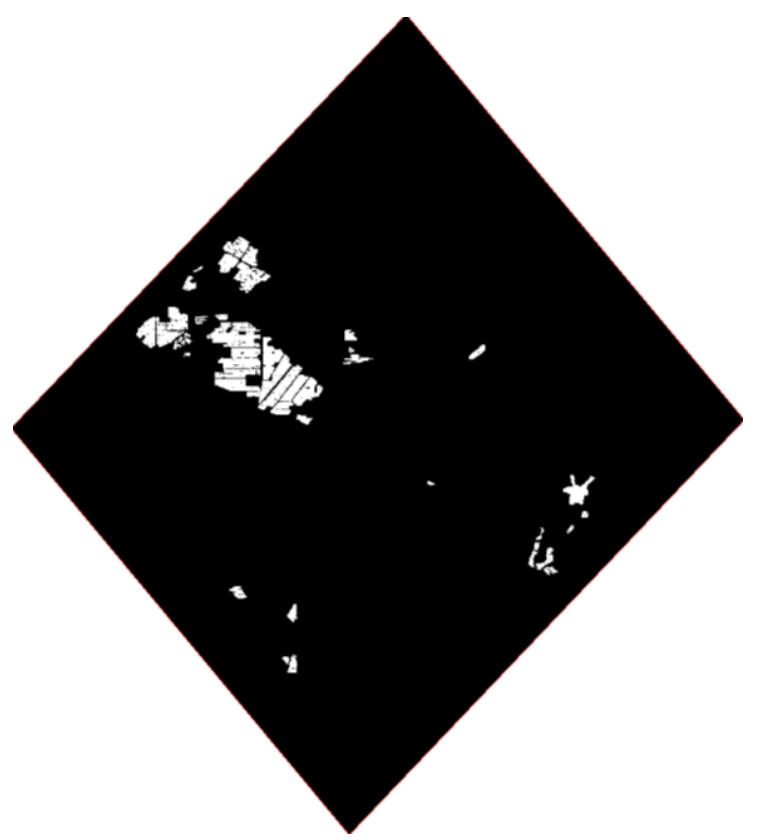

Figure 11: Target map of photovoltaic panels

The accuracy of PV panel detection is estimated using high resolution satellite images of the same area where PV panels are clearly visible. To avoid class imbalance issue, equal no. of random samples is generated through visual inspection for both positive (i.e., the class that contains PV panel) and negative class (i.e., the class that does not contain PV panel). The overall accuracy of PV panel detection is observed to be $98 \%$. A few numbers of false detection are observed in places where electrical substations are present in the scene. A high abundance value of materials of electrical substation in PV panel abundance image is quite obvious than other two material types i.e., sand and vegetation. However, an alternate methodology or output filtration may further be applied to improve the result. The spectral signature of electrical substation is given in figure 12 which is found to be distinct than the PV panel (figure 6). Hence, the spectral filtering is applied on the output and the final result is given in figure 13 .

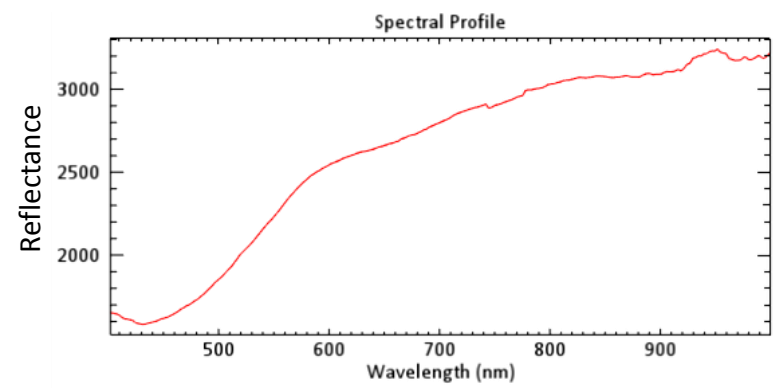

(a)

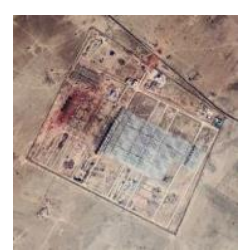

(b)

Figure 12: (a) Spectral signature of electrical substation, (b) reference HR image of the location.

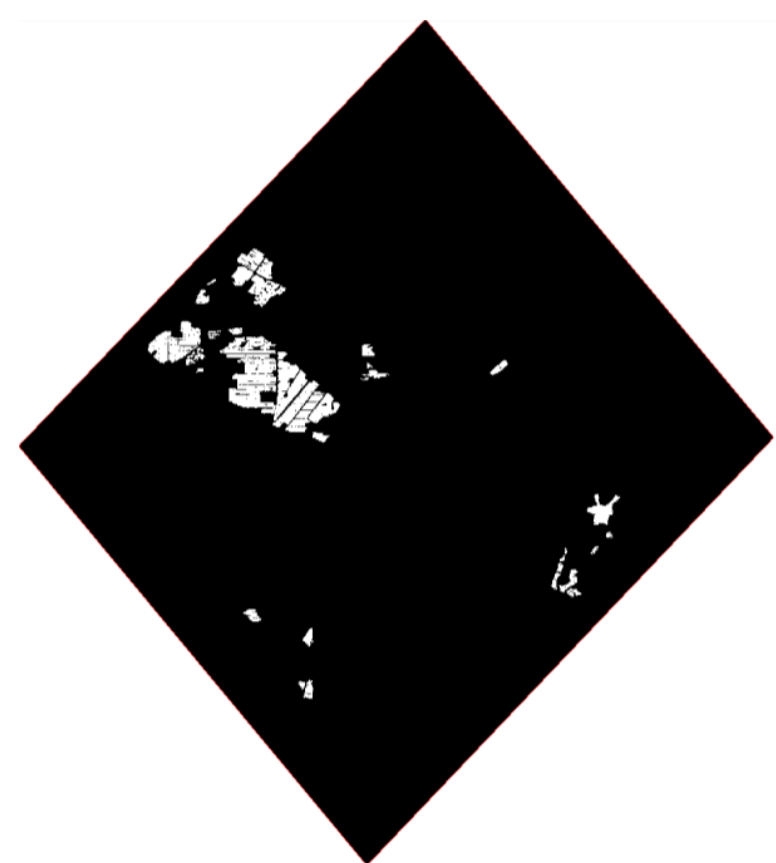

Figure 13: Target map of photovoltaic panels (after filtering)

\section{CONCLUSION}

Present work demonstrates two case studies of target detection using DESIS data. DESIS provides high spectral resolution of $2.55 \mathrm{~nm}$ in the spectral range from 400 and $1000 \mathrm{~nm}$ which is very useful in detecting objects distinctly. The first case study demonstrates the efficiency of DESIS data in detecting brick kilns in the study area with very high precision using ACE method. In the second case study, solar panels are detected using DESIS data where a linear spectral unmixing technique is employed. The accuracy of the output is observed to be very high. From the experimental results it is observed that target objects are detected very accurately using DESIS data.

\section{REFERENCES}

Alonso K. et al., Data Products, Quality and Validation of the DLR Earth Sensing Imaging Spectrometer (DESIS), Sensors 2019, 19, 4471; doi:10.3390/s19204471.

Bai X., L. Li, X. Xie, W. Li, Y. Wu and L. Gao, "FPGA Implementation for Hyperspectral Target Detection with Adaptive Coherence Estimator," 2019 IEEE International Conference on Signal, Information and Data Processing (ICSIDP), 2019, pp. 1-5, doi: 10.1109/ICSIDP47821.2019.9173104.

Bar S., Bass O., Volfman A., Dallal T., and Rotman S.R., "Geometrical interpretation of the adaptive coherence estimator for hyperspectral target detection", Proc. SPIE 8743, Algorithms and Technologies for Multispectral, Hyperspectral, and Ultraspectral Imagery XIX, 87430K, 2013, doi: $10.1117 / 12.2006472$.

Beralmar, 2011, modern technologies for the Indian brick and tile industry, 
http://www.resourceefficientbricks.org/pdf/icmb/beralmar.pdf, accessed on 29/05/2020 at 13: 49 IST.

Bhadla Solar Park, Rajasthan. Available online: https://www.nsenergybusiness.com/projects/bhadla-solar-parkrajasthan/ (accessed on 24 April 2019).

Bioucas-Dias J.M., A. Plaza, N. Dobigeon, M. Parente, Q. Du, P. Gader, and J. Chanussot, "Hyperspectral Unmixing Overview: Geometrical, Statistical, and Sparse Regression-Based Approaches," IEEE Journal of Selected Topics in Applied Earth Observations and Remote Sensing, vol. 5(2), pp. 354-379, 2012.

Bitar A. W., Jean-Philippe Ovarlez, Loong-Fah Cheong, Ali Chehab. Automatic Target Detection for Sparse Hyperspectral Images. Saurabh Prasad; Jocelyn Chanussot. Hyperspectral Image Analysis - Advances in Signal Processing and Machine Learning, Springer Nature Switzerland, pp.435-462, In press, 978-3-030-38617-7. hal-02089019v3, 2020.

Boardman, J. W. "Inversion of Imaging Spectrometry Data Using Singular Value Decomposition." In Proceedings, IGARSS'89, 12th Canadian Symposium on Remote Sensing 4 (1989): 20692072.

Boardman, J. W. "Sedimentary Facies Analysis Using Imaging Spectrometry: A Geophysical Inverse Problem." Ph.D. Thesis, University of Colorado (1992).

Boyd D. S., B. Jackson, J. Wardlaw, G. M. Foody, S. Marsh, and K. Bales, "Slavery from space: Demonstrating the role for satellite remote sensing to inform evidence-based action related to un sdg number 8," ISPRS journal of photogrammetry and remote sensing, vol. 142, pp. 380-388, 2018.

Cavalli R. M., G. A. Licciardi, and J. Chanussot, "Detection of anomalies produced by buried archaeological structures using nonlinear principal component analysis applied to airborne hyperspectral image," IEEE Journal of Selected Topics in Applied Earth Observations and Remote Sensing, vol. 6, no. 2, pp. 659-669, April 2013.

Chang C.-I, Hyperspectral data processing: Algorithm design and analysis, Wiley, 2013.

Cichocki A., R. Zdunek, A.H. Phan, and S.-I. Amari, Nonnegative matrix and tensor factorizations. Applications to exploratory multi-way data analysis and blind source separation, Wiley, Chichester, UK, 2009.

Datt B., T. R. McVicar, T. G. van Niel, D. L. B. Jupp, and J. S. Pearlman, "Preprocessing EO-1 Hyperion hyperspectral data to support the application of agricultural indexes," IEEE Transactions on Geoscience and Remote Sensing, vol. 41, pp. 1246-1259, June 2003.

eBrickkiln, 2020, http://brickkilnsoftware.net/, Accessed on 29/05/2020 at 13:17 hrs.

Eismann M. T., A. D. Stocker, and N. M. Nasrabadi, "Automated hyperspectral cueing for civilian search and rescue," Proc. IEEE, vol. 97, no. 6, pp. 1031-1055, June 2009.
Eismann M. T., A. D. Stocker, and N. M. Nasrabadi, "Automated hyperspectral cueing for civilian search and rescue," Proceedings of the IEEE, vol. 97, no. 6, pp. 1031-1055, June 2009.

Frontera-Pons J., F. Pascal, and J.-P. Ovarlez, "Adaptive nonzero-mean Gaussian detection," IEEE Transactions on Geoscience and Remote Sensing, vol. 55, no. 2, pp. 1117-1124, Feb 2017.

Heiden, U.; Müller, R. DESIS Mission. Available online: https://www.dlr.de/eoc/desktopdefault.aspx/ tabid-13614 (accessed on 15 September 2019).

Hörig B., F. Kühn, F. Oschütz, and F. Lehmann, "HyMap hyperspectral remote sensing to detect hydrocarbons," International Journal of Remote Sensing, vol. 22, pp. 1413-1422, May 2001.

Jnawali K., Kerekes J. P., Rao N., "Comparative study of spectral matched filter, constrained energy minimization, and adaptive coherence estimator for subpixel target detection based on hyperspectral imaging," Proc. SPIE 10644, Algorithms and Technologies for Multispectral, Hyperspectral, and Ultraspectral Imagery XXIV, 106441V, 2018, doi:10.1117/12.2304360.

Karoui M. S., F. z. Benhalouche, Y. Deville, K. Djerriri, X. Briottet and A. L. Bris, "Detection and Area Estimation for Photovoltaic Panels in Urban Hyperspectral Remote Sensing Data By An Original Nmf-Based Unmixing Method," IGARSS 2018 - 2018 IEEE International Geoscience and Remote Sensing Symposium, 2018, pp. 1640-1643, doi 10.1109/IGARSS.2018.8518204.

Kiln Eco, 2015, DownToEarth, 07 June, https://www.downtoearth.org.in/coverage/kiln-eco-9779, Accessed on 29/05/2020 at 13:10 hrs.

Krutz, D.; Müller, R.; Knodt, U.; Günther, B.;Walter, I.; Sebastian, I.; Säuberlich, T.; Reulke, R.; Carmona, E.; Eckardt, A.; et al. The Instrument Design of the DLR Earth Sensing Imaging Spectrometer (DESIS). Sensors 2019, 19, 1622, doi:10.3390/s 19071622 .

Limem A., G. Delmaire, M. Puigt, G. Roussel and D. Courcot, "Non-negative matrix factorization using weighted beta divergence and equality constraints for industrial source apportionment," Proc. IEEE International Workshop on Machine Learning for Signal Processing (IEEE MLSP 2013), Southampton, UK, 2013.

Maithel S., S. Kumar and D. Lalchandani, 2014, tunnel kiln technology, Factsheets about brick kilns in south and south-east Asia, https://www.shareweb.ch/site/Climate-Change-andEnvironment/about\%20us/about\%20gpcc/Documents/07\%20Tu nnel\%20Kiln.pdf, accessed on 29/05/2020 at 13:45 IST.

Malof J.M. , K. Bradbury, L.M. Collins and R.G. Newell, "Automatic detection of solar photovoltaic arrays in high resolution aerial imagery," Applied Energy, vol. 183, pp. 229$240,2016$.

Manolakis D., E. Truslow, M. Pieper, T. Cooley, and M. Brueggeman, "Detection algorithms in hyperspectral imaging systems: An overview of practical algorithms," IEEE Signal Processing Magazine, vol. 31, no. 1, pp. 24-33, Jan 2014. 
Matteoli S., M. Diani, and G. Corsini, "A tutorial overview of anomaly detection in hyperspectral images," IEEE Aerosp. Electron. Syst. Mag., vol. 25, no. 7, pp. 5-27, July 2010.

Misra P., R. Imasu, S. Hayashida, A. A. Arbain, R. Avtar and W. Takeuchi, 2020, Mapping Brick Kilns to Support Environmental Impact Studies around Delhi using Sentinel-2, ISPRS International Journal of Geo-Information, 9, 544; doi:10.3390/ijgi9090544

Müller, R.; Avbelj, J.; Carmona, E.; Gerasch, B.; Graham, L.; Günther, B.; Heiden, U.; Kerr, G.; Knodt, U.; Krutz, D.; et al. The New Hyperspectral Sensor DESIS on the Multi-Payload Platform MUSES Installed on the ISS. Int. Arch. Photogramm. Remote Sens. Spat. Inf. Sci. 2016, 41, 461-467.

Nasrabadi N. M., 2014, Hyperspectral Target Detection, IEEE Signal Processing Magazine, Jan, 34-44, DOI: 10.1109/MSP.2013.2278992.

Nazir U., U. K. Mian, M. U. Sohail, M. Taj and M. Uppal, 2020, Kiln-Net: A Gated Neural Network for Detection of Brick Kilns in South Asia, IEEE Journal of Selected Topics in Applied Earth Observations and Remote Sensing, DOI 10.1109/JSTARS.2020.3001980.

Paul A., Bhattacharya S., Dutta D., Sharma J.R., Dadhwal V. K., 2015, Band selection in hyperspectral imagery using spatial cluster mean and genetic algorithms, GIScience and Remote Sensing, 52(6):644-661. doi: 10.1080/15481603.2015.1075180.

Paul A. and Chaki N., 2020, Supervised data driven approach for hyperspectral band selection using quantization, Geocarto International, doi: 10.1080/10106049.2020.1822929.

Paul A. and Chaki N., 2021a, Dimensionality reduction using band correlation and variance measure from discrete wavelet transformed hyperspectral imagery, Annals of Data Science, 8 , 261-274, DOI: 10.1007/s40745-019-00210-x.

Paul A., Bhoumik S., 2021, Classification of hyperspectral imagery using spectrally partitioned HyperUnet, Neural Computing and Applications, doi: 10.1007/s00521-021-06532-3.

Paul A., Bhoumik S., and Chaki N., 2021, "SSNET: An improved deep hybrid network for hyperspectral image classification", Neural Computing and Applications. 33:1575-1585. DOI: 10.1007/s00521-020-05069-1.

Paul A. and Chaki N., 2019, Dimensionality reduction of hyperspectral images using pooling, Pattern Recognition and Image Analysis. 29 (1): 72-78. DOI: $10.1134 /$ S 1054661819010085

Paul A. and Chaki N., 2021b, Dimensionality Reduction of Hyperspectral image using signal entropy and spatial information in genetic algorithm with discrete wavelet transformation, Evolutionary Intelligence. 14 (4), 1793-1802, DOI: 10.1007/s12065-020-00460-2.

Paul A., Kundu A., Chaki N., Dutta D., Jha C. S., 2021b, Wavelet enabled convolutional autoencoder based deep neural network for hyperspectral image denoising, Multimedia Tools and Applications, DOI: 10.1007/s11042-021-11689-z.
Puttemans S., W.V. Ranst and T. Goedemé, "Detection of photovoltaic installations in RGB aerial imaging: A comparative study," Proc. GEOBIA 2016, Enschede, The Netherlands, 2016.

Reed I. S. and X. Yu, "Adaptive multiple-band CFAR detection of an optical pattern with unknown spectral distribution," IEEE Trans. Acoust., Speech, Signal Process., vol. 38, no. 10, pp. 1760-1770, Oct. 1990.

Robey F. C., D. R. Fuhrmann, and E. J. Kelly, “A CFAR adaptive matched filter detector," IEEE Trans. Aerosp. Electron. Syst., vol. 28, no. 1, pp. 208-216, Jan. 1992.

Scharf L. L. and B. Friedlander, "Matched subspace detectors," IEEE Trans. Signal Processing, vol. 42, no. 8, pp. 2146-2157, Aug. 1994.

Supe H. et al, Google Earth Engine for the Detection of Soiling on Photovoltaic Solar Panels in Arid Environments, Remote Sens. 2020, 12, 1466; doi:10.3390/rs12091466

Weyant C., V. Athalye, S. Ragavan, U. Rajarathnam, D. Lalchandani, S. Maithel, E. Baum, and Tami C. Bond, 2014, Environmental Science \& Technology, 48 (11): 6477-6483 DOI: $10.1021 / \mathrm{es} 500186 \mathrm{~g}$.

Yang, Shuo, and Z. Shi. "SparseCEM and SparseACE for Hyperspectral Image Target Detection. ” IEEE Geoscience and Remote Sensing Letters 11.12(2014):2135-2139. 